\title{
Late Complication of a Silicone Implant Thirty Years after Orbital Fracture Reconstruction
}

Chi An Lee,

Seok Joo Kang, Ji Young Yun, Hook Sun

Department of Plastic and Reconstructive Surgery, Busan-Baik Hospital, Inje University School of Medicine, Busan, Korea

No potential conflict of interest relevant to this article was reported.

\begin{abstract}
Alloplastic materials used for orbital fracture reconstruction can induce complications, such as infection, migration, extrusion, intraorbital hemorrhage, and residual diplopia. Silicone is one of the alloplastic materials that has been widely used for decades. The author reports a rare case of spontaneous extrusion of a silicone implant that was used for orbital fracture reconstruction 30 years earlier. A 50 -year-old man was admitted to the emergency room for an exposed substance in the lower eyelid area of the left eye, which began as a palpable hard nodule a week earlier. The exposed material was considered to be implant used for previous surgery. Under general anesthesia, the implant and parts of the fibrous capsule tissue were removed. Several factors hinder the diagnosis of implant extrusions that occur a long period after the surgery. So, surgeons must be aware that complications with implants can still arise several decades following orbital fracture reconstruction, even without specific causes.
\end{abstract}

Keywords: Silicone / Prostheses and implant / Orbital implants / Orbital fracture / Postoperative complication

\section{INTRODUCTION}

Reconstruction materials used for orbital fracture reconstruction are broadly categorized into autografts, such as bones and cartilage, and alloplastic materials, such as silicone, porous polyethylene, metallic mesh, hydroxyapatite, and polylactide. Reconstructions using alloplastic materials are becoming growingly preferred over autograft implantation as they do not require additional harvesting [1]. However, alloplastic materials can also induce complications such as infection, migration, extrusion, intraorbital hemorrhage, and residual diplopia [2]. The author reports a rare case of spontaneous extrusion of a silicone implant that was used for orbital fracture reconstruction 30 years earlier.

Correspondence: Seok Joo Kang

Department of Plastic and Reconstructive Surgery, Busan Baik Hospital, Inje University School of Medicine, 75 Bokji-ro, Busanjin-gu, Busan 47392, Korea

E-mail: sonydr@naver.com

Received February 28, 2017 / Revised May 14, 2017 / Accepted May 15, 2017

\section{CASE REPORT}

A 50-year-old man was admitted to the emergency room for an exposed substance in the lower eyelid area of the left eye, which began as a palpable hard nodule a week earlier. The patient had a history of orbital wall reconstruction to the same eye, via the subcilliary approach, 30 years previously. He had no particular post-

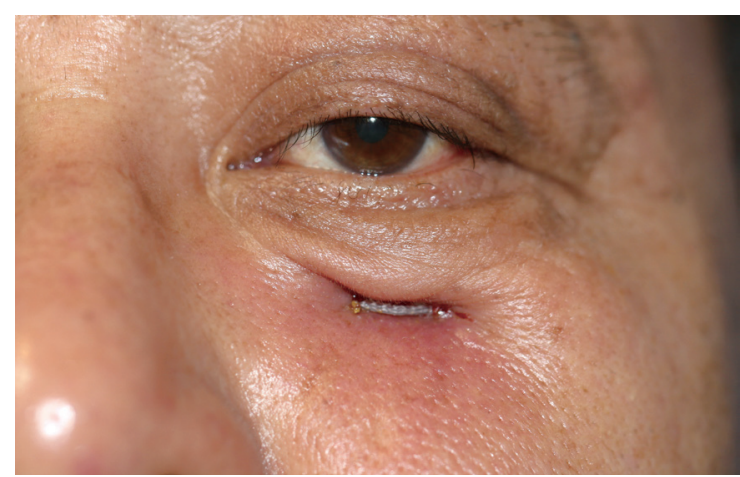

Fig. 1. Case. Partial exposure of the silicone implant material used for orbital wall reconstruction is seen, with surrounding swelling and redness. 
operative complication, unusual trauma history, or signs of infection such as redness or pain, until the appearance of the nodule.

Orbital examination revealed mild swelling and redness around the exposed substance, and a well-healed subcilliary incision. The exposed material was $1 \mathrm{~cm}$ in length, $0.3 \mathrm{~cm}$ in width, and was suspected to be the silicone implant located approximately $1.5 \mathrm{~cm}$ inferior to the lower eyelid (Fig. 1). Despite mild proptosis on the left cheek, functional test results of the extraocular muscles were normal, and no diplopia was present in any field of gaze. A computed tomography (CT) scan revealed an old blowout fracture in the medial and inferior walls of the left orbit, with an augmented implant and dense, soft tissue that had thickened to about $7 \mathrm{~mm}$ above the implant (Fig. 2).

Under general anesthesia, the skin around the exposed implant was incised and dissected to expose the orbital floor. It was observed that the implant was surrounded by a dense fibrous tissue that was firmly attached to the orbital floor within the bony defect. There was no pus or severely inflamed tissue (Fig. 3). The implant and parts of the fibrous capsule tissue were eliminated, leaving the superior dome attached to the tissue of the orbital floor, including the periosteum. Additional reconstruction of the orbital wall was not performed due to the intact orbital floor, and the absence of significant enophthalmos (Figs. 4, 5). Postoperative recovery was uneventful, and the patient was discharged 4 days after surgery.

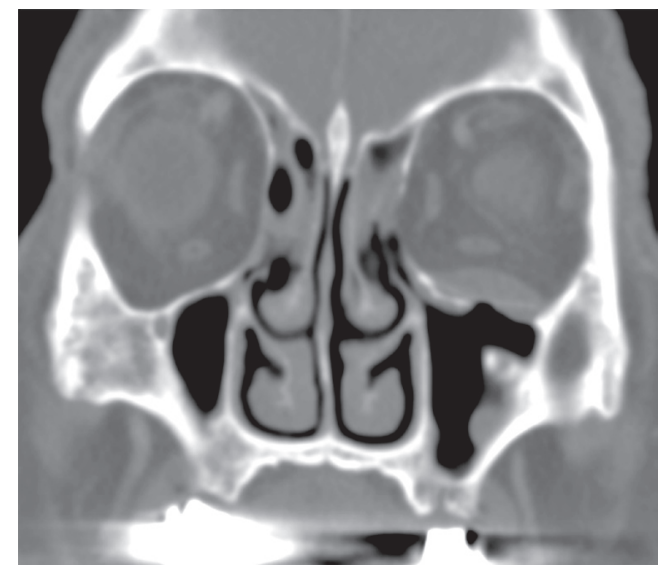

Fig. 2. Preoperative computed tomography scan. Inserted implant material is visualized at the bone defect region of the orbital floor. Enlarged soft tissue is observed around the implant.

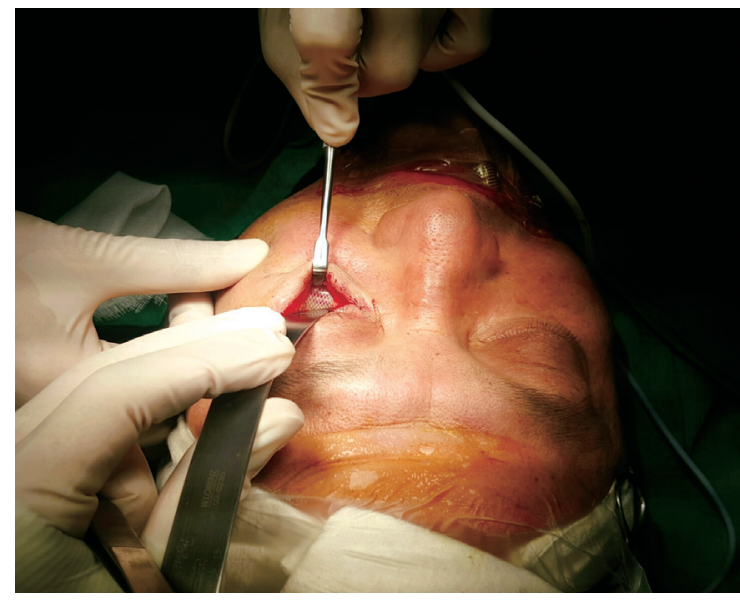

Fig. 3. Intraoperative photograph. Approach through the skin around the extruded wound. There were no visible signs of abscess or significant inflammation.

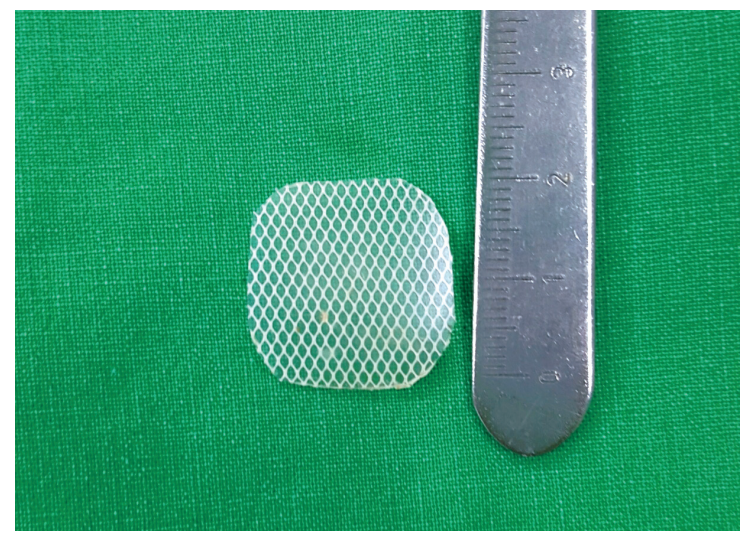

Fig. 4. Removed implant. A $2 \mathrm{~cm} \times 2 \mathrm{~cm}$-sized silicone implant was removed.

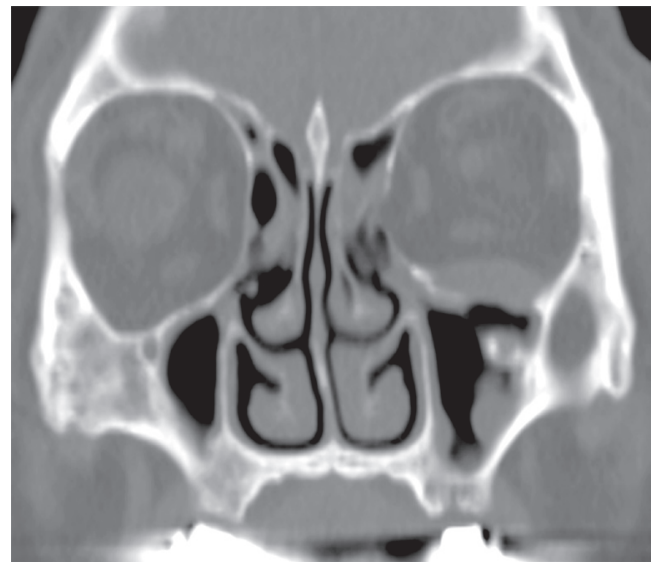

Fig. 5. Postoperative computed tomography scan. Postoperative state of the implant removal; the orbital barrier is maintained as per the preoperative state. 


\section{DISCUSSION}

Blow-out fractures are a common type of craniofacial fracture, usually caused by trauma, falling, traffic accident, or direct contusion. A blow-out fracture causes various functional and cosmetic complications which require treatment, such as periorbital edema, sensory impairment, extraocular muscle limitation, diplopia, and enopthalmos [3]. The goal for surgical treatment is to reconstruct the orbital wall and secure the intraorbital structure and volume [4]. Alloplastic materials, suchas silicone, porous polyethylene (Medpor), metallic mesh, hydroxyapatite, and polylactide are commonly used for orbital wall reconstruction. Use of alloplastic materials can reduce the time required for the procedure, eliminate donor-site morbidity, and the materials are supplied in multitudes of sizes and shapes. Silicone is a popular choice because it is inexpensive, easy to handle, and chemically inert. In addition, it is noncarcinogenic, and can be sterilized [5,6].

However, there are also disadvantages to silicone implants. Complications such as postoperative infection, migration, or extrusion to the dermis or sinus, and residual diplopia have been reported [7]. Smooth silicone implants have been reported to cause extrusion in $3.1 \%$ of cases, complications related to infections in $1.2 \%$ of cases, displacement in $2 \%$ of cases, and seroma in $0.5 \%$ of cases. Overall, the removal rate due to complications associated with silicone implants for orbital wall reconstruction has been reported to be $13 \%[6]$.

This is the first case of an implant extrusion occurring 30 years after surgery with no known cause. The exact reasons for spontaneous protrusion following orbital wall reconstruction are unclear, but fibrous capsules have been proposed as the cause, wherein the collagen of the capsule contracts and causes the implant to migrate to a less resistant area [1]. In addition, earlier surgical techniques that did not stabilize the implant may have contributed to the implant migration [6].

Diagnosis of implant extrusions occurring a long period after the surgery is difficult for several reasons. The implant is not palpable until it has migrated to areas near the skin, and the migration is largely asymptomatic. Obtaining the procedural history is not an option due to the long time interval. Also, even if the patient can provide a history, medical records are unobtainable. The most effective method for diagnosis would be to observe the implant, the orbit, the sinus, and surrounding tissues via CT images. In this way, the CT findings could be correlated with the patient's symptoms and signs before making a diagnosis. Once the diagnosis is made, removal of the implant is sufficient treatment, and if there is no inflammation the fibrous capsule (which forms a barrier to the orbital content) does not need to be completely removed [1]. For this patient, the 'superior dome' made by the fibrous capsule and periosteum on the orbital floor (as observed by CT scan) was spared as much as possible after removal of the implant and the excessive fibrous tissue.

Several factors hinder the diagnosis of implant extrusions that occur a long period after the surgery. First, the implant is not palpable until it migrates to areas near the skin, and it is largely asymptomatic. Second, history taking regarding surgery is not an option due to the long gap in time following the surgery. Third, even if a history is taken from the patient, medical records are unobtainable.

In conclusion, surgeons should be aware that complications with implants can still arise several decades following orbital fracture reconstruction, even without specific causes. Various complications may arise from the implant because of the complex periorbital neural, vascular, and muscular structures. To prevent implant migration, the implant could be fixed or porous implant could be used; the pores are known to provide stability that prevents migration and exposure [6]. Furthermore, surgeons should also explain to the patient who receives an orbital implant that there is a possibility of long-term complications. These patients need to be made aware that if they experience any symptoms associated with the eye with the reconstructed orbit, even after several decades, they should immediately seek medical attention.

\section{REFERENCES}

1. Warrier S, Prabhakaran VC, Davis G, Selva D. Delayed complications of silicone implants used in orbital fracture repairs. Orbit 2008;27:147-51.

2. Su Y, Sun J, Fan X. Epithelial cysts associated with alloplastic implants 
after repair of orbital fractures: a systematic review and four new cases. Br J Oral Maxillofac Surg 2016;54:658-63.

3. Dedhia R, Tollefson TT. Delayed periorbital abscess after silicone implant to orbital floor fracture. Craniomaxillofac Trauma Reconstr 2016;9:185-7.

4. Nam SB, Bae YC, Moon JS, Kang YS. Analysis of the postoperative outcome in 405 cases of orbital fracture using 2 synthetic orbital implants. Ann Plast Surg 2006;56:263-7.
5. Prowse SJ, Hold PM, Gilmour RF, Pratap U, Mah E, Kimble FW. Orbital floor reconstruction: a case for silicone. A 12 year experience. J Plast Reconstr Aesthet Surg 2010;63:1105-9.

6. Potter JK, Ellis E. Biomaterials for reconstruction of the internal orbit. J Oral Maxillofac Surg 2004;62:1280-97.

7. Klisovic DD, Katz SE, Lubow M. The wayward implant: orbital silicone plate extrusion associated with squamous epithelial downgrowth and infection. Orbit 2002;21:149-54. 